\title{
Cannabis, a potential treatment option in pediatric IBD? Still a long way to go
}

\section{Nienke Halbmeijer, Michael Groeneweg \& Lissy De Ridder}

To cite this article: Nienke Halbmeijer, Michael Groeneweg \& Lissy De Ridder (2019) Cannabis, a potential treatment option in pediatric IBD? Still a long way to go, Expert Review of Clinical Pharmacology, 12:4, 355-361, DOI: 10.1080/17512433.2019.1582330

To link to this article: https://doi.org/10.1080/17512433.2019.1582330

Accepted author version posted online: 15

Feb 2019.

Published online: 26 Feb 2019.

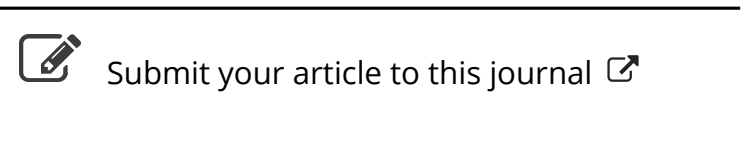

Џll Article views: 76

View Crossmark data ¿ 


\title{
Cannabis, a potential treatment option in pediatric IBD? Still a long way to go
}

\author{
Nienke Halbmeijer ${ }^{\mathrm{a}}$, Michael Groeneweg ${ }^{\mathrm{a}}$ and Lissy De Ridder ${ }^{\mathrm{b}}$ \\ aDepartment of Pediatrics, Maasstad Hospital, Rotterdam, The Netherlands; 'bepartment of Paediatric Gastroenterology, Erasmus MC-Sophia \\ Children's Hospital, Rotterdam, The Netherlands
}

\section{ABSTRACT}

Introduction: The onset of inflammatory bowel disease (IBD) in children is rising. Current treatment options are based on immunomodulatory therapy. Alternative treatment options are upcoming since they appear to be effective in individual patients. Cannabis might relief IBD symptoms in these cases and improve quality of life. Recent evidence suggests a potential anti-inflammatory effect of cannabis. Areas covered: This review presents an overview of recent literature on the use of cannabis in IBD focussing on pediatric IBD patients. Background information on the role of the endocannabinoid system within the gastrointestinal tract is presented. Other modalities of cannabis and its purified ingredients will be discussed as well, with attention to its applicability in children with IBD.

Expert opinion: More research is needed on the efficacy and safety of cannabis in pediatric IBD. Studies are well underway, but until then the use of cannabis in pediatric IBD cannot be recommended.
ARTICLE HISTORY

Received 4 May 2018

Accepted 11 February 2019

KEYWORDS

Adolescents; cannabis; cannabidiol; children; endocannabinoid system; inflammatory bowel disease; medical marijuana; pediatric

\section{Introduction}

The incidence of pediatric inflammatory bowel disease (IBD) is steeply rising globally [1]. IBD includes Crohn's disease and ulcerative colitis, both diseases affect the gastroin testinal tract by immune-mediated inflammation. These diseases are characterized by relapsing and remitting episodes of active disease, which may be accompanied by abdominal pain, nausea, loss of appetite and diarrhea. The current treatment options are based on the reduction of inflammatory processes, by using immunomodulatory therapies, such as corticosteroids, thiopurines, methotrexate and/or TNF-a blockers. Despite the improvement in therapy, many IBD patients report ongoing symptoms (abdominal pain, fatigue, poor appetite) with significant impact on quality of life. Cannabis has been studied as an alternative treatment to relief IBD symptoms. Over the past decades, cannabis or also known as medical marijuana has gained more acceptance for conditions like chemotherapy-related nausea and vomiting and chronic pain but also for spasticity and tremor in neurological diseases. Cannabis may be promising in improving the quality of life of IBD patients, by ameliorating symptoms like: abdominal pain, nausea, diarrhea, and poor appetite. Recent evidence suggests there also might be a potential anti-inflammatory effect of cannabis on IBD disease activity. So cannabis is of high interest for this medical condition.

Population-based surveys show that many adult and adolescent IBD patients use cannabis to relieve abdominal symptoms [2-6]. About $10-20 \%$ of patients are still under the age of 18 years when diagnosed with IBD $[1,7]$. In this population, $32 \%$ of the IBD patients use cannabis [8]. As a result, pediatric gastroenterologists will more frequently be confronted with patients who will use cannabis to relieve IBD-related symptoms. There fore, we conducted a review to summarize the present scientific evidence about the efficacy and safety of cannabis in the treatment of pediatric IBD.

For this review, a literature search was performed in Pubmed and EMBASE on trials who evaluated the effect of cannabis on IBD until September 2018. Keywords used were 'inflammatory bowel diseases,' 'Crohn disease,' 'Ulcerative colitis,' 'Cannabis,' 'Medical Marijuana,' and 'Cannabinoids.'

\section{The endocannabinoid system and the gastrointestinal tract}

The effect of cannabis on the gastrointestinal tract is based on involvement of the endocannabinoid system in gut functioning. The endocannabinoid system contains receptors, endogenous cannabinoids, synthesizing and metabolizing enzymes that are active in both the central and peripheral nervous system but also in many other organs. They modulate neuronal signaling and influence physiological processes as sleep, mood and emotion, appetite, pain perception and memory [9]. Two endogenous ligands of the endocannabinoid system have been discovered: anandamide (AEA) and 2-arachidonoylglycerol (2-AG). Both ligands are responsible for endocannabinoid signaling. They are inhibited by their metabolizing enzymes, fatty acid amine hydrolase (FAAH) and monoacylglycerol lipase (MAGL), respectively. The most thoroughly investigated part of the endocannabinoid system is the cannabinoid 1 and 2 (CB1 and CB2) receptor, which are widely distributed in the gastrointestinal tract. They are found in the enteric nervous system and epithelial cells. Particularly CB1 receptors are expressed in the mucosa of the stomach and colon. In 
Article highlights

- Incidence of pediatric IBD is rising, while conventional treatment in many patients fails. Alternative treatment options have to be explored.

- The endocannabinoid system is involved in gut homeostasis and can be altered in an inflamed gastrointestinal tract.

- Questionnaires in adolescent and young-adult IBD patients report relieve of IBD symptoms and improved quality of life when using cannabis.

- Cannabis seems to have a potential immunomodulating effect on IBD in murine colitis models.

- Preclinical data suggest a relevant role for endocannabinoiddegrading enzymes, such as FAAH and MAGL. To what extent inhibitors of these enzymes will cause decreased inflammation in humans needs to be further investigated.

- Until there is sufficient data about the efficacy and safety profile of cannabis, cannabis use needs to be actively discouraged in children with IBD.

- Further research needs to focus on clinical response and evaluation of mucosal healing due to cannabis or its purified ingredients.

contrast, in non-inflamed human intestinal epithelium, CB2 receptors are absent or only weakly expressed $[10,11]$. CB2 receptors are mainly expressed by immune cells (neutrophils, plasma cells, activated macrophages and subsets of $B$ and $T$ cells) and to a lesser extent by enteric neurons [11]. Activation of CB1 receptors leads to relaxation of smooth muscle and to decreased gut motility and gut secretion. Based on the current knowledge, the endocannabinoid system as a whole is seen as a physiological entity that controls gut homeostasis [12-14].

In addition, the endocannabinoid system has a role in gut inflammation. The endocannabinoid system probably serves as an endogenous gastrointestinal defense system by affecting the regulation of the endogenous cannabinoids. Furthermore, inhibition of the metabolizing enzymes (FAAH, MAGL) ensures ongoing activity of the endocannabinoids ligands ( $A E A$ and 2-AG) and $C B$ receptors [15]. Activation of $C B 2$ receptors causes suppression of inflammation, resulting in improvement of mucosal healing in murine colitis models [15-17]. So, potentially the endocannabinoid system is a target for new therapies in IBD.

\subsection{Role of cannabis on gastrointestinal inflammation}

Recent evidence suggests that an anti-inflammatory effect can be attributed to cannabis [14,18-20]. Cannabis contains phytocannabinoids $\Delta$ 9-tetrahydrocannabinol (THC) and cannabidiol (CBD). THC is known as a partial agonist of CB1 and CB2 receptors. By activating $C B$ receptors, THC suppresses the production of pro-inflammatory cytokines and chemokines and induces $T$ cell apoptosis [15,20]. $\Delta$ 9-tetrahydro cannabinolic acid (THCA), a precursor of THC, has antiinflammatory activity on colon epithelial cell cultures, by inhibiting IL-8 secretion [19]. Animal models show that cannabidiol is capable of modulating the endocannabinoid system, through activation of CB1 by inhibiting FAAH [21]. However, because of the complex pathogenesis of IBD it remains uncertain whether these anti-inflammatory effects can be expected in humans as well.

\section{Cannabis use among adult patients with IBD}

The literature search identified 80 publications. Trials and surveys on cannabis treatment for IBD were included. Only one article focussed on adolescent and pediatric IBD patients. Studies performed in adults were included as well. By handsearching, we finally included nine studies that evaluate cannabis treatment for adult patients with IBD [2-6,22-25] and one for adolescent IBD patients (13-21 years of age) [8]. These studies are shown in Table 1. In summary, a high prevalence of cannabis use in adult and adolescent IBD patients is seen in various countries [2-6]. Many patients report beneficial effects of cannabis on IBD-related symptoms, as well as improvement of the quality of life. Only one study group presented their results between Crohn's disease and ulcerative colitis patients separately [22]. The ulcerative colitis-group consisted of two patients so these patients were precluded from statistical analysis. More objective measurements were obtained with the Crohn's Disease Activity Index (CDAl) and Harvey Bradshaw score, which significantly decreased after a period of cannabis use [22-24]. This effect was not seen with cannabidiol [25]. A few side effects (panic attack, drowsiness, poor diet, lethargy, and addiction) of cannabis were reported by patients [3]. However, the safety profile of cannabis and cannabidiol in the treatment of IBD is poorly examined. Until now, cannabis in the treatment of IBD seems promising.

Clear limitations of these studies were the low number of included patients and the lack of standardization of the used amount or the route of use of cannabis. No studies report an endoscopic or histological evaluation of disease activity before and after cannabis use. Therefore, the occurrence of mucosal healing in human intestinal epithelium is unknown. Based on current research no distinction can be made between the effects of cannabis use on symptom relief in Crohn's disease or ulcerative colitis. Crohn's disease is caused by transmural inflammation instead of ulcerative colitis where the inflammation is limited to the mucosa, with the result that abdominal pain is more frequently seen in Crohn's disease. This distinction could be relevant in further research. The high prevalence rates of cannabis in IBD patients may be influenced by the recent approval of medical marijuana by various states in the US and Canada and also by increased attention in the press about the beneficial effects of cannabis use for medical purposes.

\section{The endocannabinoid system and the brain}

The endocannabinoid system is not only involved in the gastrointestinal tract. The CB1 receptors are also widely distributed in the brain [26], which explains psychotropic properties as described in cannabis use and abuse.

The endocannabinoid system is already present in the early stages of brain development. During embryonal development of the central nervous system, CB1 receptors and endogenous cannabinoids ( $A E A$ and 2-AG) were detectable from 14 weeks of gestation [27]. The endocannabinoid system is subject to constant change from childhood to adolescence [27-29]. Therefore, cannabis use may have an adverse effect on brain development, in case of early exposure, making cannabis a controversial treatment option in children. Studies show that 


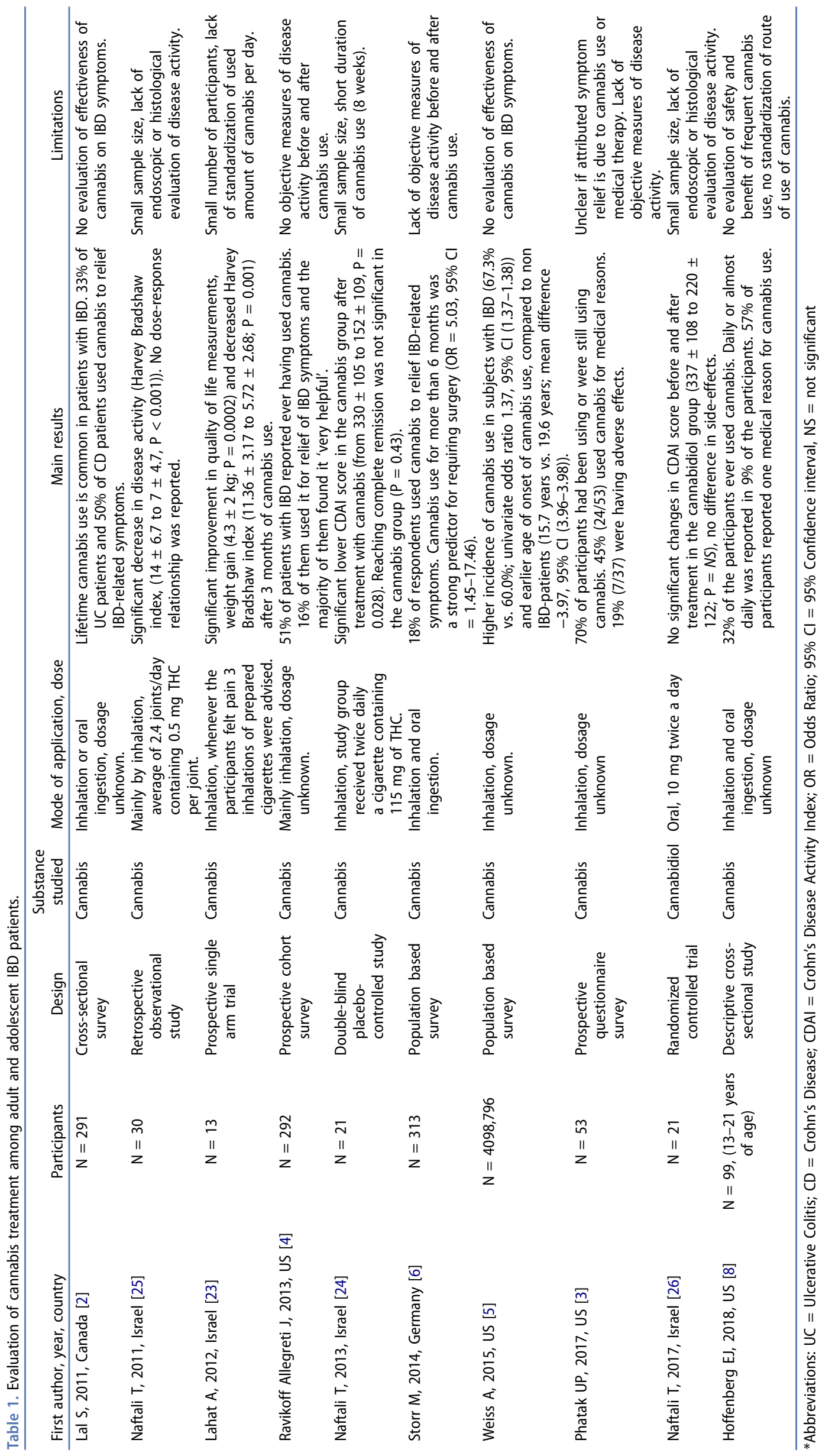


adolescents who frequently use cannabis in high doses have a lower intelligence quotient (IQ) and reduced cognitive function [30]. These effects are dose-dependent. Cessation of cannabis use for 1 year does not result in full recovery of neurocognitive functioning, especially when cannabis abuse was started in adolescence $[30,31]$. The precise extent to which cannabis itself is responsible for these effects is unknown. Lower social-economic status and young age may influence the observed effect on cognitive function [32].

THC is the primary psychoactive component of cannabis and is able to affect brain function, by influencing cognitive functions (learning, working memory and attention). Also neurophysiologic effects can occur, symptoms are: anxiety, paranoia and psychiatric disorders (hallucinations, schizophrenia, and schizophrenia-like psychotic disorders) [33,34]. A dose-response relationship is seen for these side-effects [35]. Regular cannabis use in high dose before the age of 15 years is associated with psychiatric disease and cognitive impairment [36].

\section{Alternative agents influencing the endocannabinoid system}

Inhalation by smoking is the most frequently used mode of application for cannabis consumption. Various side-effects and risk for developing an addiction to smoking are well-known. Therefore, other modes of application are urgently needed.

\subsection{Cannabidiol oil}

One of the treatment options is cannabidiol (CBD) oil. It is important to acknowledge that $C B D$ has low affinity for the $\mathrm{CB} 1$ and $\mathrm{CB} 2$ receptors resulting in the absence of psychotropic effects [21,37]. Therefore, CBD could be used safely in children with IBD. However, a low dose of $20 \mathrm{mg} /$ day of CBD does not improve Crohn's Disease Activity Index (CDAI) of adults with IBD. This lack of effect on IBD symptoms can be explained by the fact that only a single compound of cannabis (only CBD) is used. The authors also suggested that the dose of CBD used might be too low to be effective [25].

CBD has successfully been used in children for therapyresistant epilepsy (Dravet syndrome, Sturge-Weber syndrome and Lennox-Gastaut syndrome), resulting in a significant reduction in seizure frequency [38-40]. One hundred and twenty children and young adults with Dravet syndrome were included in a double-blind, placebo-controlled trial that reported a decrease of median seizure frequency per month from 12.4 to $5.9(95 \% \mathrm{Cl}-41.1$ to $-5.4 ; \mathrm{P}=0.01)$ during the 14 week treatment period [38]. This reduction was also seen in a double-blind, placebo-controlled trial in 225 children and adults with Lennox-Gastaut syndrome after 14 weeks of CBD use $(41.9 \%$ reduction in the $20 \mathrm{mg} / \mathrm{kg} C B D$ group $(P=0.005)$ compared to $37.2 \%$ reduction in the $10 \mathrm{mg} / \mathrm{kg}$ CBD group ( $\mathrm{P}=$ 0.002)) [40]. Four children with Sturge-Weber syndrome showed a significant drop in seizure frequency after 8 weeks of CBD treatment (average number of seizures per month dropped from 10.7 to 7.7) [39]. The dose used was between 5 and $25 \mathrm{mg} / \mathrm{kg} /$ day orally. Kaplan et al. [39] started with a low dose of CBD (5 mg/kg/day) and increased the dose by $5 \mathrm{mg} /$ $\mathrm{kg} /$ day per week as tolerated up to a maximum of $25 \mathrm{mg} / \mathrm{kg} /$ day. Continuous doses of $10 \mathrm{mg} / \mathrm{kg} /$ day or $20 \mathrm{mg} / \mathrm{kg} /$ day were used by Devinsky et al. $[38,40]$. More adverse effects were seen when higher doses (20-25 mg/kg/day) of CBD were used: somnolence, lethargy, behavioral problems, and increased seizure activity. In addition, gastrointestinal adverse effects were reported (diarrhea and vomiting) in $45 \%$ of the patients with Dravet syndrome and in $16-26 \%$ of patients with LennoxGastaut syndrome. After dose reduction, almost all adverse effects resolved completely. $0.05-0,13 \%$ of the patients who received $C B D$ had to be withdrawn from the trial and discontinued $C B D$ administration due to adverse effects. Laboratory values showed elevated liver enzymes in the CBD group $[38,40]$.

\subsection{Sativex ${ }^{\circledast}$ (Nabiximols)}

Sativex is an oromucosal plant-based cannabinoid spray. It is mainly used in patients with multiple sclerosis where it is able to relieve spasticity-related and neuropathic pain [41]. Also patients with chronic pain and post-operative pain experience beneficial effects of Sativex. A meta-analysis on the efficacy of cannabis-based medicines, including Sativex, for pain management, showed a significant pain reduction in chronic pain $-0.61(-0.78$ to $-0.43, P<0.0001)$ [42]. This spray contains both phytocannabinoids THC and CBD. Each spray delivers a dose of $100 \mu \mathrm{L}$, containing $2.7 \mathrm{mg}$ THC and $2.5 \mathrm{mg}$ CBD. Sativex is generally well tolerated in patients with multiple sclerosis-related spasticity; however, adverse effects (such as anxiety, depression, constipation or diarrhea, nausea, headache, dry mouth and somnolence) were commonly seen. These effects occurred in $<10 \%$ of people and usually appear during the first 4 weeks of treatment. More serious effects (allergic phenomena, euphoria, psychosis, panic, hallucinations, paranoia, suicidal ideation, memory changes, and cognitive decline) were seen in $<1 \%$ of people [41]. No clinical trials have been performed on the efficacy and safety of Sativex in patients with IBD.

\subsection{Synthetic cannabinoids}

Nabilone and Dronabinol are synthetic cannabinoids, both can be administered orally. They mimic the effect of THC by acting like $C B$ receptor agonists and alter endocannabinoid signaling. Both synthetic cannabinoids are approved for the treatment of nausea and vomiting caused by chemotherapy in cancer patients. Dronabinol is also approved as an appetite stimulant in AIDS patients with weight loss. Nabilone was studied as an alternative antiemetic in children with chemotherapy for cancer. The authors conducted a double-blind crossover study in 23 patients (aged from 1 to 17) with nabilone compared to domperidone. In the nabilone group adverse effects were more frequent, most commonly reported were drowsiness (55\%), dizziness (36\%) and mood changes (14\%). One patient experienced hallucinations and withdrew from the trial. For the other participants, adverse effects were thought to be acceptable, they continued the treatment [43]. The effect of nabilone and dronabinol on IBD symptoms and mucosal inflammation has not been studied. 


\subsection{FAAH and MAGL inhibitors}

Another pharmacological strategy is to continue endocannabinoid signaling by inhibiting the metabolizing enzymes of anandamide (AEA) and 2-arachidonoylglycerol (2-AG), fatty acid amine hydrolase (FAAH) and monoacylglycerol lipase (MAGL), respectively, instead of direct activation of the CB receptors [44]. Selective inhibition of FAAH or FAAH blockade reduced damage in experimental colitis models [14,45]. Also, MAGL inhibitors have potential to counteract colitis and related systemic inflammation [46]. So, both agents seem to have anti-inflammatory effects in murine colitis models. For the time being there are no studies in IBD patients with FAAH or MAGL inhibitors. Further research on this topic is highly recommended because of the potential immunomodulating mechanism.

\section{Cannabis for medical purposes in children}

Various effects of unintentional cannabis ingestion in children have been described such as lethargy, ataxia, hypotonia, mydriasis, tachycardia, hypoventilation and even respiratory insufficiency [47]. Despite these adverse effects, would cannabis be a potential treatment option in pediatric IBD?

A recent descriptive cross-sectional study in Colorado, described cannabis use in 99 pediatric patients (aged between 13 and 22 years) with IBD. As mentioned in the introduction, 32\% of them used cannabis once during their lifetime while $9 \%$ used cannabis daily or almost daily. Most frequently medical purposes for using cannabis were reported. Other reasons were to relax, to relieve tension, to feel good and to have a good time with friends. Participants used cannabis by smoking cigarettes or ingesting edibles. However, $37 \%$ reported problems with consuming cannabis like craving and need for higher doses to reach the same effect with the risk of developing addiction [8].

Pediatric IBD patients might benefit by relief in abdominal pain, nausea, cramping, diarrhea, and improvement in appetite and weight gain. These benefits are thought to be the result of the effect of cannabis on the endocannabinoid system and associated anti-inflammatory effect. However, the mechanism of action of THC and cannabidiol is not fully clarified, and well-designed clinical trials have not been performed. As a result of symptom reduction without an actual decrease of inflammation the IBD disease activity may be underestimated by the patient, causing higher risks of serious complications. Side-effects remain a problem in children as well as concerns regarding brain development and cognition.

The mode of application is another important issue in children. Smoking cannabis clearly is an unacceptable option in children, because of the well-known negative effects of tobacco smoking on health. Therefore, oral cannabidiol (like the oral mucosal spray Sativex ${ }^{\oplus}$ ) would be the preferred mode of application. However, no studies on the pharmacokinetics and pharmacodynamics of cannabidiol in children have been performed.

\section{Conclusion}

In summary, cannabis might potentially be a treatment option in pediatric IBD in addition to conventional treatment. However, to what extent cannabis acts as an immunomodulating agent or just enhances a general feeling of well-being is unclear currently. First, we support well-designed clinical trials to evaluate the efficacy and safety of oral cannabidiol in children with IBD. These trials should include clinical response but also evaluate mucosal healing. For this specific age group, extra focus has to be on the mode of application. Awaiting future research, cannabis should actively be discouraged as a treatment modality in pediatric IBD.

\section{Expert opinion}

During the last decade, IBD in children has a rising incidence and is accompanied by a high disease burden. Since conventional therapies regularly are not sufficient, patients and parents are looking for alternative treatment options. Currently, cannabis is of high interest in this field. Evidence suggests that cannabis relieves IBD symptoms, improves quality of life and to a lesser extent induces immunomodulating effects. However, according to our literature research, we advise against the prescription of cannabis in pediatric IBD. There are still a lot of questions that need to be answered first.

The first key question is: what is the actual impact of cannabis on inflammation in IBD? Preclinical data in murine colitis models suggest that activation of $C B$ receptors may lead to mucosal healing by inhibiting the inflammatory reaction $[10,15]$. Direct activation of the $C B$ receptors may be caused by inhalation of cannabis, using purified ingredients (such as CBD and Sativex ${ }^{\oplus}$ ) or synthetic cannabinoids (Nabilone and Dronabinol). Moreover, continuous high levels of endogenous cannabinoids will lead to activation of the $C B$ receptors as well. This effect can be achieved by inhibitors of the endocannabinoid-degrading enzymes, FAAH and MAGL. So far, these concepts are only tested in experimental trials. Whether activation of the $C B$ receptors will realize mucosal healing in $I B D$ patients as well, need to be further investigated. Therefore, it is important to evaluate disease activity endoscopically before and after treatment with one of these agents.

Second key question is which agent would be most applicable in pediatric patients. As mentioned earlier smoking cigarettes is no option in this category of patients. Purified ingredients of cannabis, therefore, are of special interest in children. Most experience exists with cannabidiol in children with therapyresistant epilepsy syndromes [38-40]. A major benefit of cannabidiol is the lack of psychotropic side-effects. Furthermore, CBD seems well tolerated in this patient population, nevertheless adverse events on behavior and gastrointestinal tract are described as well. An anti-inflammatory effect of CBD on IBD in humans is not proved yet. Experimental studies in animals have shown promising results of immunomodulating effects of CBD on colitis models [48]. An already available THC/CBD solution is the oromucosal spray, Sativex ${ }^{\oplus}$, which appears to be easily suitable for pediatric IBD patients, though a serious doubt about sativex is the presence of THC in the solution. Due to the known effects of THC on the central nervous system, psychotropic adverse effect can be experienced by patients. More experience in children exists with Nabilone, a synthetic cannabinoid. Nabilone was used in children with chemotherapy-related nausea as an antiemetic agent. However, serious side-effects like hallucinations were experienced by one of the patients [43]. 
Consequently, nabilone is likely not suitable for the treatment of pediatric IBD. Furthermore, long-term effects of cannabidiol and Sativex ${ }^{\circledR}$ are unknown. Chronic use of cannabis is associated with impaired neurodevelopment outcome and addiction. Whether these agents can cause these serious adverse effects as well is unclear. The same is probably true for synthetic cannabinoids through the equal mechanism of action in the endocannabinoid system.

Knowledge about the endocannabinoid system is rapidly expanding. With the increasing availability of legal cannabis and medical marijuana around the world, the use of cannabis for medical purposes is of high interest. There still are many gaps in understanding these cannabinoid-based medicines before they can be administrated to children, so further research in this field is sorely needed. Currently, the main benefit of cannabis for the treatment of IBD is improvement of quality of life which is most likely caused by a general wellbeing, a known effect of cannabis. To what extent this effect can be attributed to reduced inflammation is uncertain.

Nowadays, the endocannabinoid system serves as an encouraging therapeutic target for various abnormal conditions of the gastrointestinal tract. The focus for the next five years should be on the influence of the endocannabinoid system on inflammation of the bowel. To evaluate whether cannabis indeed can induce mucosal healing in the human gut, endoscopic examination before and after treatment is needed. The existing preclinical trials in murine colitis models report a major role for the endogenous cannabinoids and its inhibitory enzymes. However, before implementation of these agents in the treatment of IBD patients further preclinical research is required to better understand the mechanism of action of the FAAH and MAGL inhibitors on gastrointestinal inflammation.

There is an urgent need for larger clinical trials. Especially elucidation of the efficacy and safety profile of cannabis and the purified ingredients is required. In our opinion, wellconducted trials on oral cannabidiol in pediatric IBD would be of high interest. The research in this field is well underway, but more time is needed before cannabis as part of IBD treatment in children can be recommended.

\section{Funding}

This paper was not funded.

\section{Declaration of interest}

$\mathrm{L}$ de Ridder discloses that for the last 3 years they have received consultation fee, research grant, or honorarium from ZonMw (national health institute), Janssen, Pfizer, Mundipharma, Shire and Abbvie. The authors have no other relevant affiliations or financial involvement with any organization or entity with a financial interest in or financial conflict with the subject matter or materials discussed in the manuscript apart from those disclosed.

\section{Reviewer disclosures}

Peer reviewers on this manuscript have no relevant financial or other relationships to disclose.

\section{References}

Papers of special note have been highlighted as either of interest $(\cdot)$ or of considerable interest (••) to readers.

1. Benchimol El, Fortinsky KJ, Gozdyra P, et al. Epidemiology of pediatric inflammatory bowel disease: a systematic review of international trends. Inflamm Bowel Dis. 2011 Jan;17(1):423-439. PubMed PMID: 20564651

2. Lal S, Prasad N, Ryan M, et al. Cannabis use amongst patients with inflammatory bowel disease. Eur J Gastroenterol Hepatol. 2011 Oct;23(10):891-896. PubMed PMID: 21795981; eng.

3. Phatak UP, Rojas-Velasquez D, Porto A, et al. Prevalence and patterns of marijuana use in young adults with inflammatory Bowel Disease. J Pediatr Gastroenterol Nutr. 2017 Feb;64(2):261-264. PubMed PMID: 27846066; eng.

- Study showing high prevalence rates of cannabis use in young adults with IBD.

4. Ravikoff Allegretti J, Courtwright A, Lucci M, et al. Marijuana use patterns among patients with inflammatory bowel disease. Inflamm Bowel Dis. 2013 Dec;19(13):2809-2814. PubMed PMID: 24185313; PubMed Central PMCID: PMCPMC4126607. eng.

5. Storr M, Devlin S, Kaplan GG, et al. Cannabis use provides symptom relief in patients with inflammatory bowel disease but is associated with worse disease prognosis in patients with Crohn's disease. Inflamm Bowel Dis. 2014 Mar;20(3):472-480. PubMed PMID: 24407485; eng.

6. Weiss A, Friedenberg F. Patterns of cannabis use in patients with inflammatory Bowel Disease: A population based analysis. Drug Alcohol Depend. 2015 Nov 1;156:84-89. PubMed PMID: 26422462; eng.

7. Kelsen J, Baldassano RN. Inflammatory bowel disease: the difference between children and adults. Inflamm Bowel Dis. 2008 Oct;14 (Suppl 2):S9-S11. PubMed PMID: 18816756; eng.

8. Hoffenberg EJ, McWilliams SK, Mikulich-Gilbertson SK, et al. Marijuana use by adolescents and young adults with inflammatory Bowel Disease. J Pediatr. 2018 Aug;199:99-105. PubMed PMID: 29673723; eng.

- Survey about adolescents and young adults with IBD who use cannabis to relieve IBD symptoms.

9. Greineisen WE, Turner H. Immunoactive effects of cannabinoids: considerations for the therapeutic use of cannabinoid receptor agonists and antagonists. Int Immunopharmacol. 2010 May;10 (5):547-555. PubMed PMID: 20219697; PubMed Central PMCID: PMCPMC3804300. eng.

10. Wright K, Rooney N, Feeney M, et al. Differential expression of cannabinoid receptors in the human colon: cannabinoids promote epithelial wound healing. Gastroenterology. 2005 Aug;129 (2):437-453. PubMed PMID: 16083701; eng.

11. Wright KL, Duncan M, Sharkey KA. Cannabinoid CB2 receptors in the gastrointestinal tract: a regulatory system in states of inflammation. Br J Pharmacol. 2008 Jan;153(2):263-270. PubMed PMID: 17906675; PubMed Central PMCID: PMCPMC2219529. eng.

12. Schicho R, Storr M. Alternative targets within the endocannabinoid system for future treatment of gastrointestinal diseases. Can J Gastroenterol. 2011 Jul;25(7):377-383. PubMed PMID: 21876860; PubMed Central PMCID: PMCPMC3174079. eng.

13. Goyal H, Singla U, Gupta U, et al. Role of cannabis in digestive disorders. Eur J Gastroenterol Hepatol. 2017 Feb;29(2):135-143. PubMed PMID: 27792038; eng.

14. Hasenoehrl C, Taschler U, Storr M, et al. The gastrointestinal tract a central organ of cannabinoid signaling in health and disease. Neurogastroenterol Motil Off J Eur Gastrointestinal Motil Soc. 2016 Dec;28(12):1765-1780. PubMed PMID: 27561826; PubMed Central PMCID: PMCPMC5130148. eng.

15. Gyires K, Zadori ZS. Role of cannabinoids in gastrointestinal mucosal defense and inflammation. Curr Neuropharmacol. 2016;14 (8):935-951. PubMed PMID: 26935536; PubMed Central PMCID: PMCPMC5333598. eng.

.. Excellent review about how the cannabinoid system is involved in the gastrointestinal mucosal defense system and might change in inflammation. 
16. Massa F, Storr M, Lutz B. The endocannabinoid system in the physiology and pathophysiology of the gastrointestinal tract. J Mol Med (Berlin, Germany). 2005 Dec;83(12):944-954. PubMed PMID: 16133420; eng.

17. Leinwand $\mathrm{KL}$, Gerich ME, Hoffenberg EJ, et al. Manipulation of the endocannabinoid system in colitis: a comprehensive review. Inflamm Bowel Dis. 2017 Feb;23(2):192-199. PubMed PMID: 28079617; eng.

18. Hasenoehrl C, Storr M, Schicho R. Cannabinoids for treating inflammatory bowel diseases: where are we and where do we go? Expert Rev Gastroenterol Hepatol. 2017 Apr;11(4):329-337. PubMed PMID: 28276820; PubMed Central PMCID: PMCPMC5388177. eng.

19. Nallathambi R, Mazuz M, lon A, et al. Anti-inflammatory activity in colon models is derived from Delta9-Tetrahydrocannabinolic Acid that interacts with additional compounds in cannabis extracts. Cannabis Cannabinoid Res. 2017;2(1):167-182. PubMed PMID: 29082314; PubMed Central PMCID: PMCPMC5627671. eng.

20. Ahmed W, Katz S. Therapeutic use of cannabis in inflammatory Bowel Disease. Gastroenterol Hepatol. 2016 Nov;12(11):668-679. PubMed PMID: 28035196; PubMed Central PMCID: PMCPMC5193087. eng.

21. Esposito G, Filippis DD, Cirillo $C$, et al. Cannabidiol in inflammatory bowel diseases: a brief overview. Phytother Res. 2013 May;27 (5):633-636. PubMed PMID: 22815234; eng.

22. Lahat A, Lang A, Ben-Horin S. Impact of cannabis treatment on the quality of life, weight and clinical disease activity in inflammatory bowel disease patients: a pilot prospective study. Digestion. 2012;85(1):1-8. PubMed PMID: 22095142; eng.

23. Naftali T, Bar-Lev Schleider L, Dotan I, et al. Cannabis induces a clinical response in patients with Crohn's disease: a prospective placebo-controlled study. Clinical gastroenterology and hepatology. Off Clin Pract J Am Gastroenterol Assoc. 2013 Oct;11 (10):1276-1280. e1. PubMed PMID: 23648372; eng.

- First placebo-controlled study that reports benefit from cannabis in IBD patients.

24. Naftali T, Lev LB, Yablecovitch D, et al. Treatment of Crohn's disease with cannabis: an observational study. Isr Med Assoc J. 2011 Aug;13 (8):455-458. PubMed PMID: 21910367; eng.

25. Naftali T, Mechulam R, Marii A, et al. Low-dose cannabidiol is safe but not effective in the treatment for Crohn's Disease, a randomized controlled trial. Dig Dis Sci. 2017 Jun;62 (6):1615-1620. PubMed PMID: 28349233; eng.

- Randomized controlled trial on oral cannabidiol for the treatment of Crohn's disease, without beneficial effects for IBD patients.

26. Pertwee RG. Pharmacology of cannabinoid CB1 and CB2 receptors. Pharmacol Ther. 1997;74(2):129-180. PubMed PMID: 9336020; eng.

27. Biegon A, Kerman IA. Autoradiographic study of pre- and postnatal distribution of cannabinoid receptors in human brain. Neurolmage. 2001 Dec;14(6):1463-1468. PubMed PMID: 11707102; eng.

28. Fride $E$. The endocannabinoid-CB receptor system: importance for development and in pediatric disease. Neuro Endocrinol Lett. 2004 Feb-Apr;25(1-2):24-30. PubMed PMID: 15159678; eng.

.. Good overview of the transformation of the endocannabinoid-CB-receptor system in perinatal development.

29. Fride E, Gobshtis N, Dahan H, et al. The endocannabinoid system during development: emphasis on perinatal events and delayed effects. Vitam Horm. 2009;81:139-158. PubMed PMID: 19647111; eng.

30. Camchong J, Lim KO, Adverse KS. Effects of cannabis on adolescent brain development: a longitudinal study. Cereb Cortex. 2017 Mar 1;27(3):1922-1930. PubMed PMID: 26912785

31. Meier MH, Caspi A, Ambler A, et al. Persistent cannabis users show neuropsychological decline from childhood to midlife. Proc Natl Acad Sci U S A. 2012 Oct 2;109(40):E2657-64. . PubMed PMID: 22927402; PubMed Central PMCID: PMCPMC3479587. eng.

32. Zucker RA, Gonzalez R, Feldstein Ewing SW, et al. Assessment of culture and environment in the adolescent brain and cognitive development study: rationale, description of measures, and early data. Dev Cogn Neurosci. 2018 Aug;32:107-120. PubMed PMID: 29627333; eng.

33. Mandelbaum DE, de la Monte SM. Adverse structural and functional effects of marijuana on the brain: evidence reviewed. Pediatr Neurol. 2017 Jan;66:12-20. PubMed PMID: 27789118; eng.

34. Volkow ND, Swanson JM, Evins AE, et al. Effects of cannabis use on human behavior, including cognition, motivation, and psychosis: a review. JAMA psychiatry. 2016 Mar;73(3):292-297. PubMed PMID: 26842658; eng.

35. Moore TH, Zammit S, Lingford-Hughes A, et al. Cannabis use and risk of psychotic or affective mental health outcomes: a systematic review. Lancet (London, England). 2007 Jul 28;370(9584):319-328. PubMed PMID: 17662880; eng

36. Lubman DI, Cheetham A, Yucel M. Cannabis and adolescent brain development. Pharmacol Ther. 2015 Apr;148:1-16. PubMed PMID: 25460036; eng.

37. Mechoulam R, Hanus L. Cannabidiol: an overview of some chemical and pharmacological aspects. Part I: chemical aspects. Chem Phys Lipids. 2002 Dec 31;121(1-2):35-43. PubMed PMID: 12505688; eng.

38. Devinsky O, Cross JH, Laux L, et al. Trial of cannabidiol for drug-resistant seizures in the dravet syndrome. $\mathrm{N}$ Engl J Med. 2017 May 25;376(21):2011-2020. PubMed PMID: 28538134; eng.

39. Kaplan EH, Offermann EA, Sievers JW, et al. Cannabidiol treatment for refractory seizures in sturge-weber syndrome. Pediatr Neurol. 2017 Jun;71:18-23. e2. PubMed PMID: 28454984; eng.

40. Devinsky O, Patel AD, Cross JH, et al. Effect of cannabidiol on drop seizures in the lennox-gastaut syndrome. N Engl J Med. 2018 May 17;378(20):1888-1897. PubMed PMID: 29768152; eng.

41. Giacoppo S, Bramanti P, Mazzon E. Sativex in the management of multiple sclerosis-related spasticity: an overview of the last decade of clinical evaluation. Mult Scler Relat Disord. 2017 Oct;17:22-31. PubMed PMID: 29055461; eng.

42. Aviram J, Samuelly-Leichtag G. Efficacy of cannabis-based medicines for pain management: a systematic review and meta-analysis of randomized controlled trials. Pain Physician. 2017 Sep;20(6): E755-e796. PubMed PMID: 28934780; eng.

43. Dalzell AM, Bartlett $H$, Lilleyman JS. Nabilone: an alternative antiemetic for cancer chemotherapy. Arch Dis Child. 1986 May;61 (5):502-505. PubMed PMID: 3013104; PubMed Central PMCID: PMCPMC1777777. eng.

- Important study of nabilone in children used as antiemetic for cancer chemotherapy which reports adverse effects, such as hallucination.

44. Salaga M, Sobczak M, Fichna J. Inhibition of fatty acid amide hydrolase (FAAH) as a novel therapeutic strategy in the treatment of pain and inflammatory diseases in the gastrointestinal tract. Eur J Pharm Sci. 2014 Feb 14;52:173-179. PubMed PMID: 24275607; eng.

45. Salaga M, Mokrowiecka A, Zakrzewski PK, et al. Experimental colitis in mice is attenuated by changes in the levels of endocannabinoid metabolites induced by selective inhibition of fatty acid amide hydrolase (FAAH). J Crohn's Colitis. 2014 Sep;8(9):998-1009. PubMed PMID: 24530133; PubMed Central PMCID: PMCPMC4136976. eng.

46. Alhouayek M, Lambert DM, Delzenne NM, et al. Increasing endogenous 2-arachidonoylglycerol levels counteracts colitis and related systemic inflammation. FASEB J. 2011 Aug;25 (8):2711-2721. PubMed PMID: 21551239; eng.

.. Interesting study showing increased levels of 2-AG are able to reduce inflammation in experimental colitis models.

47. Richards JR, Smith NE, Moulin AK. Unintentional cannabis ingestion in children: A systematic review. J Pediatr. 2017 Nov;190:142-152. PubMed PMID: 28888560; eng.

48. De Filippis D, Esposito G, Cirillo C, et al. Cannabidiol reduces intestinal inflammation through the control of neuroimmune axis. PLoS one. 2011;6(12):e28159. PubMed PMID: 22163000; PubMed Central PMCID: PMCPMC3232190. eng. 